Pacific Journal of Mathematics

SPECTRAL DISTRIBUTION OF THE SUM OF SELF-ADJOINT 


\title{
SPECTRAL DISTRIBUTION OF THE SUM OF SELF-ADJOINT OPERATORS
}

\author{
ARTHUR LIEBERMAN
}

Using the techniques of noncommutative integration theory, classical results of Hermann Weyl concerning the positive eigenvalues of the sum of two self-adjoint compact operators are extended to self-adjoint operators which are measurable with respect to a gage space. Let $(H, A, m)$ be a gage space and let $K$ and $L$ be self-adjoint operators which are measurable with respect to $(H, A, m)$. Let $P_{K}[\lambda, \infty)$ be the spectral projection of $K$ for the interval $[\lambda, \infty)$ and let $\Lambda_{K}(x)=\sup \left\{\lambda \mid m\left(P_{E}[\lambda, \infty)\right) \geqq x\right\}$. Then $\Lambda_{K+L}(x+r) \leqq \Lambda_{K}(x)+$ $A_{L}(r)$. If $K \leqq L$, then $\Lambda_{K}(x) \leqq \Lambda_{L}(x)$. If $L$ is bounded, then $\Lambda_{L K L}(x) \leqq\|L\|^{2} A_{K}(x)$ for $x \leqq m\left(P_{K}[0, \infty)\right)$. If $q=m$ (support $(L))$ and $q<\infty$, then $\Lambda_{K}(x+q) \leqq \Lambda_{K+L}(x)$; if $\mu=\Lambda_{|K|}(q)$, then $\|K+L\|_{p} \geqq\left\|K P_{K}(-\mu, \mu)\right\|_{p}$ for $1 \leqq p \leqq \infty$.

1. Notation. We specifically work in the context of a gage space. [See 5 for definitions and notation.] We will always require that an operator be measurable [5, Definition 2.1]. This is a technical consideration which is necessary to avoid the pathologies which can occur with unbounded operators. Any one of the following conditions implies that a self-adjoint operator $T$ is measurable with respect to the gage space $(H, A, m)$ :

1. $T \in A$.

2. $\operatorname{T\eta } A$ and $m$ is a finite gage. ( $T \eta A$ means that $U T=T U$ for every unitary operator $U$ in the commutant of $A$.)

3. $T \eta A$ and $m$ (support $(T))<\infty$, where support $(T)$ is the orthocomplement of the nullspace of $T$.

4. $T \eta A$ and $A$ is abelian.

If $P$ is a projection operator, $P$ will be identified with the range of $P$. If $T$ is an operator, $R(T)$ denotes the range of $T$ and $\bar{R}(T)$ denotes the closure of $R(T)$. If $T$ is self-adjoint, note that support $(T)=\bar{R}(T)$.

$(H, A, m)$ is a gage space. If $S$ and $T$ are self-adjoint operators which are measurable with respect to $(H, A, m)$, then $S+T(S T)$ will denote the strong sum (product) of $S$ with $T$; this is the closure of the ordinary sum (product) and is self-adjoint and measurable [5, Corollary 5.2]. $T$ has spectral decomposition $T=\int_{-\infty}^{\infty} \lambda d P_{T}(\lambda)$; the function $P_{T}(\lambda)$ is chosen to be continuous from the right. If $\mathscr{J}$ is an interval, $P_{T}(\mathscr{F})$ is the spectral projection of $T$ for the interval $\mathscr{F}$. The function $\Lambda_{T}$ is defined, for $x>0$, by $\Lambda_{T}(x)=\sup \left\{\lambda \mid m\left(P_{T}[\lambda, \infty)\right) \geqq x\right\}$. 
Note that $m\left(P_{T}\left[\Lambda_{T}(x), \infty\right)\right)<x$ is possible if $m\left(P_{T}\left(\Lambda_{T}(x)-\varepsilon, \Lambda_{T}(x)\right)\right)=\infty$ for every $\varepsilon>0 . \Lambda_{T}(x)$ is a nonincreasing function of $x$ and is continuous from the left. If $x>m(I)$, where $I$ is the identity operator on $H$, then $A_{T}(x)=\sup (\dot{\rho})=-\infty$; we will not explicitly mention this pathology in order to avoid excessive technicality and splitting into cases.

The author wishes to thank the referee for finding an error in one of the proofs and for suggestions which make the paper more readable.

2. Statement of the results. Below we state the theorems and corollaries and prove the corollaries. The theorems are proved in the next section. For the remainder of this paper, $K$ and $L$ are self-adjoint operators which are measurable with respect to the gage space $(H, A, m)$.

THEOREM 1. Let $q=m$ (support $(L)$ ) and assume $q<\infty$. Then $\Lambda_{K}(x+q) \leqq \Lambda_{K+L}(x)$ for $x>0$; equivalently, $\Lambda_{K}(x) \leqq \Lambda_{K+L}(x-q)$ for $x>q$.

THEOREM 2. $\Lambda_{K+L}(x+r) \leqq \Lambda_{K}(x)+\Lambda_{L}(r)$, for $x>0, r>0$.

If $(H, A, m)$ is the algebra of all bounded operators on $H, m$ is the usual trace, and $K$ is a compact operator and has (counting multiplicity) at least $j+1$ positive eigenvalues, then $\Lambda_{K}(j)$ is the $j$ th positive eigenvalue of $K$ and $\Lambda_{K}(j+1 / 2)$ is the $(j+1)$ st positive eigenvalue of $K$. If in addition $L$ is compact and has at least $k+1$ positive eigenvalues, then :Theorem 2 implies $\Lambda_{K+L}(j+k+1) \leqq$ $\Lambda_{K}(j+1 / 2)+\Lambda_{L}(k+1 / 2)=\Lambda_{K}(j+1)+\Lambda_{L}(k+1)$, which is Weyl's result [6, Satz 1]. Similarly, Theorem 1 reduces to [6, Satz 2] if $L$ has finite rank.

By $K \leqq L$ is meant $L-K \geqq 0$.

Corollary 1 . If $K \leqq L$, then $\Lambda_{K}(x) \leqq \Lambda_{L}(x)$ for $x>0$.

Proof of Corollary 1. $K=L+(K-L)$. Note that $K-L \leqq 0$. Let $x>0$ and let $\varepsilon$ be an arbitrary positive number with $\varepsilon<x$. Then $\Lambda_{K}(x) \leqq \Lambda_{L}(x-\varepsilon)+\Lambda_{K-L}(\varepsilon)$ by Theorem 2 . Since $\Lambda_{K-L}(\varepsilon) \leqq 0$ and the function $A_{L}$ is continuous from the left, $\Lambda_{K}(x) \leqq \Lambda_{L}(x)$.

COROLlary 2. If $K \leqq L$ and $f$ is a nondecreasing real-valued function with domain $(-\infty, \infty)$, then $m(f(K)) \leqq m(f(L))$, provided these quantities are both defined.

Proof of Corollary 2. $\quad m(f(K))=\int_{-\infty}^{\infty} f(\lambda) d m\left(P_{K}(\lambda)\right)$ and $m(f(L))=$ 
$\int_{-\infty}^{\infty} f(\lambda) d m\left(P_{L}(\lambda)\right)$. The conclusion is immediate since by Corollary 1 , $m\left(P_{K}[\lambda, \infty)\right) \leqq m\left(P_{L}[\lambda, \infty)\right)$ and $m\left(P_{L}(-\infty, \lambda]\right) \leqq m\left(P_{K}(-\infty, \lambda]\right)$ for all real numbers $\lambda$. Note that the hypotheses of Corollary 2 do not imply $f(K) \leqq f(L)[1]$.

THEOREM 3. $\Lambda_{|K+L|}(x+r) \leqq \Lambda_{|K|}(x)+\Lambda_{|L|}(r)$ for $x>0$ and $r>0$, where $|K|$ is the absolute value of the operator $K$.

Corollary 3. Let $q=m($ support $(L))$ and assume $q<\infty$. Then $\Lambda_{|K|}(x+q) \leqq \Lambda_{|K+L|}(x)$ for $x>0$; equivalently, $\Lambda_{|K|}(x) \leqq \Lambda_{|K+L|}(x-q)$ for $x>q$.

Proof of Corollary 3. Let $x>0$, and let $\varepsilon$ be an arbitrary positive number with $\varepsilon<x$. Since $K=(K+L)-L$, by Theorem 3 , $\Lambda_{|K|}(x+q) \leqq \Lambda_{|K+L|}(x-\varepsilon)+\Lambda_{|L|}(q+\varepsilon)=\Lambda_{|K+L|}(x-\varepsilon)$ since $\Lambda_{|L|}(q+\varepsilon)=0$ or $-\infty$. Now apply the left continuity of the function $\Lambda_{|K+L|}$.

Theorem 4. Assume $L$ is bounded with norm $\|L\|$. Then $\Lambda_{L K L}(x) \leqq\|L\|^{2} \Lambda_{K}(x)$ for $0<x \leqq m\left(P_{K}[0, \infty)\right)$. In particular, if $P$ is a projection in $A$, then $\Lambda_{P_{K} P}(x) \leqq \Lambda_{K}(x)$ for $0<x \leqq m\left(P_{K}[0, \infty)\right)$.

CoRollary 4. Assume $L$ is invertible and $a I \leqq|L| \leqq b I$ for some positive numbers $a$ and $b$. Then $a^{2} \Lambda_{K}(x) \leqq \Lambda_{L K L}(x) \leqq b^{2} \Lambda_{K}(x)$ for $0<x \leqq m\left(P_{K}[0, \infty)\right)$, where $I$ is the identity operator on $H$.

Proof of Corollary 4. Clearly $|L| \leqq b$ and $\left|L^{-1}\right| \leqq 1 / a$. Apply Theorem 4 to $L K L$ and to $L^{-1}(L K L) L^{-1}=K$.

The $p$-norm of a self-adjoint measurable operator $T$ is defined [3, Definition 3.1] by $|T|_{p}=\left(m\left(|T|^{p}\right)\right)^{1 / p}$ if $1 \leqq p<\infty$ and $\|T\|_{\infty}=$ $\sup \left\{\lambda \mid m\left(P_{|T|}[\lambda, \infty)\right)>0\right\}$. Note that $\|T\|_{\infty}$ equals the operator norm of $T$ if the gage space is regular, that is, if the gage of every nonzero projection is positive.

THEOREM 5. Let $q=m$ (support $(L))$ and assume $q<\infty$. Let $\mu=\Lambda_{|K|}(q)$. Then $\|K+L\|_{p} \geqq\left\|K P_{K}(-\mu, \mu)\right\|_{p}$ for $1 \leqq p \leqq \infty$.

\section{Proof of the theorems.}

Lemma 1. Let $P$ and $Q$ be projections in $A$. Let $Y=\{v \in H \mid P v=v$ and $Q v=0\}$. Then $Y \in A$ and $Y+\bar{R}(P Q)=P$.

Proof of Lemma 1. Let $v \in Y$ and $w \in R(P Q), w=P Q z$. Then 
$\langle v, w\rangle=\langle v, P Q z\rangle=\langle P v, Q z\rangle=\langle v, Q z\rangle=\langle Q v, z\rangle=\langle 0, z\rangle=0$, so that $Y \perp \bar{R}(P Q)$.

Now let $P y=y$. Let $z$ be the projection of $y$ on the subspace $\bar{R}(P Q)$. Then $y=(y-z)+z$. Clearly $z \in \bar{R}(P Q)$ and consequently, $P(y-z)=y-z$. In addition, $(y-z) \perp \bar{R}(P Q)$. Let $w$ be any vector. Then $\langle Q(y-z), w\rangle=\langle Q P(y-z), w\rangle=\langle y-z, P Q w\rangle=0$, so that $Q(y-z)=0$.

Lemma 2. Let $P$ and $Q$ be projections in $A$. Let $Y=\{v \in H \mid P v=v$ and $Q v=0\}$. Then $m(Y) \geqq m(P)-m(Q)$. In particular, if $P v=v$ implies $Q v \neq 0$, then $m(Q) \geqq m(P)$.

Proof of Lemma 2. By additivity of the gage and Lemma 1, $m(Y)+m(\bar{R}(P Q))=m(P)$. To prove the lemma, it suffices to show that $m(\bar{R}(P Q)) \leqq m(Q)$; this is well-known for factors but we know of no reference for the general case. For later use, this is proved under the assumption that $P$ is self-adjoint but is not necessarily a projection.

The operator $P Q$ has polar decomposition [2, pp. 323-324] $P Q=$ $M\left(Q P^{2} Q\right)^{1 / 2}$, where $M$ is a partial isometry with initial domain support $\left(Q P^{2} Q\right)^{1 / 2}=$ support $\left(Q P^{2} Q\right)=\bar{R}\left(Q P^{2} Q\right)$ and terminal domain support $(P Q P)^{1 / 2}=\operatorname{support}(P Q P)=\bar{R}(P Q P)$. Consequently, $\bar{R}(P Q)=\bar{R}(P Q P)$; also $m(\bar{R}(P Q P))=m(\bar{R}(Q P Q))$ since the initial domain and the terminal domain of a partial isomerty have the same gage. Then $m(\bar{R}(P Q))=$ $m(\bar{R}(P Q P))=m(\bar{R}(Q P Q)) \leqq m(Q)$ since $\bar{R}(Q P Q)$ is a subspace of $Q$.

If $H$ is finite dimensional, Lemma 2 states that the dimension of the solution space of a system of $m(Q)$ homogeneous linear equations in $m(P)$ unknowns is at least $m(P)-m(Q)$.

Proof of Theorem 1. Let $x>0$ and $\varepsilon>0$ and $\mu=\Lambda_{K}(x+q)$. Then $m\left(P_{K}[\mu-\varepsilon, \infty)\right) \geqq x+q$. Apply Lemma 2 with $P=P_{K}[\mu-\varepsilon, \infty)$ and $Q=$ support $(L)$ to obtain $m\left\{v \in H \mid P_{K}[\mu-\varepsilon, \infty) v=v\right.$ and $L v=$ $0\} \geqq(x+q)-q=x$. If $P_{K}[\mu-\varepsilon, \infty) v=v$ and $L v=0$, then $\langle(K+$ $L) v, v\rangle \geqq(\mu-\varepsilon)\|v\|^{2}$, so that $P_{K+L}[\mu-\varepsilon, \infty) v \neq 0$. By Lemma 2, $m\left(P_{K+L}[\mu-\varepsilon, \infty)\right) \geqq m\left\{v \in H \mid P_{K}[\mu-\varepsilon, \infty) v=v\right.$ and $\left.L v=0\right\} \geqq x$. Since $\varepsilon$ is an arbitrary positive number, $\Lambda_{K+L}(x) \geqq \mu=\Lambda_{K}(x+q)$.

Proof of Theorem 2. Let $x>0$ and $r>0$, and assume $\Lambda_{K+L}(x+$ $r)>\Lambda_{K}(x)+\Lambda_{L}(r)$. Let $4 \delta=\Lambda_{K+L}(x+r)-\Lambda_{K}(x)-\Lambda_{L}(r)$. Let $P=$ $P_{K+L}\left[\Lambda_{K+L}(x+r)-\delta, \infty\right)$; then $m(P) \geqq x+r$. Let $Q$ be projection on the subspace of $H$ spanned by $P_{K}\left[\Lambda_{K}(x)+\delta, \infty\right)$ and $P_{L}\left[\Lambda_{L}(r)+\delta, \infty\right)$; then $m(Q)<x+r$.

Let $P v=v$ with $\|v\|=1$. Then $\langle(K+L) v, v\rangle \geqq \Lambda_{K+L}(x+r)-$ $\delta=\Lambda_{K}(x)+\Lambda_{L}(r)+4 \delta-\delta>\left(\Lambda_{K}(x)+\delta\right)+\left(\Lambda_{L}(r)+\delta\right)$, so that either 
$\langle K v, v\rangle>\Lambda_{K}(x)+\delta$ or $\langle L v, v\rangle>\Lambda_{L}(r)+\delta$. Therefore $Q v \neq 0$. By Lemma $2, m(Q) \geqq m(P)$, which is impossible since $m(P) \geqq x+r$ and $m(Q)<x+r$.

Proof of Theorem 3. Let $\lambda>0$ and $\psi>0$. Apply Lemma 2 with $P=P_{K+L}[\lambda+\psi, \infty)$ and $Q=$ projection on the subspace of $H$ spanned by $P_{K}[\lambda, \infty)$ and $P_{L}[\psi, \infty)$ to obtain $m\left(P_{K+L}[\lambda+\psi, \infty)\right) \leqq$ $m(Q) \leqq m\left(P_{K}[\lambda, \infty)\right)+m\left(P_{L}[\psi, \infty)\right)$. Similarly, $m\left(P_{-(K+L)}[\lambda+\psi, \infty)\right) \leqq$ $m\left(P_{-K}[\lambda, \infty)\right)+m\left(P_{-L}[\psi, \infty)\right)$. Adding these inequalities yields $m\left(P_{|K+L|}[\lambda+\psi, \infty)\right) \leqq m\left(P_{|K|}[\lambda, \infty)\right)+m\left(P_{|L|}[\psi, \infty)\right)$.

Let $\delta$ be a small positive number. Then $m\left(P_{|K+L|}\left[\Lambda_{|K|}(\lambda)+\delta+\right.\right.$ $\left.\left.\Lambda_{|L|}(\psi)+\delta, \infty\right)\right) \leqq m\left(P_{|K|}\left[\Lambda_{|K|}(\lambda)+\delta, \infty\right)\right)+m\left(P_{|L|}\left[\Lambda_{|L|}(\psi)+\delta, \infty\right)\right)<$ $\lambda+\psi$, so that $\Lambda_{|K+L|}(\lambda+\psi)<\Lambda_{|K|}(\lambda)+\Lambda_{|L|}(\psi)+2 \delta$.

Proof of Theorem 4. Without loss of generality assume $\|L\|=1$. We will show that $m\left(P_{L K L}[\lambda, \infty)\right) \leqq m\left(P_{K}[\lambda, \infty)\right)$ for $\lambda>0$. Let $\lambda>0$ and let $P=P_{L K L}[\lambda, \infty)$. Let $v \in H,\|v\|=1$, and $P v=v$. Then $\langle L K L v, v\rangle \geqq \lambda$, so that $\langle K L v, L v\rangle \geqq \lambda$. Since $\|L v\| \leqq 1, P_{K}[\lambda, \infty) L v \neq 0$ and $L P_{K}[\lambda, \infty) L v \neq 0$. Let $Q$ be projection on $\bar{R}\left(L P_{K}[\lambda, \infty) L\right)$; then $Q v \neq 0$. By Lemma $2, m(P) \leqq m(Q)$. But $m(Q) \leqq m\left(\bar{R}\left(L P_{K}[\lambda, \infty)\right)\right.$ because of set inclusion, and $m\left(\bar{R}\left(L P_{K}[\lambda, \infty)\right) \leqq m\left(P_{K}[\lambda, \infty)\right)\right.$; this is proved in the last paragraph of the proof of Lemma 2 .

Proof of Theorem 5. If $p=\infty,\|K+L\|_{\infty}=\lim _{\varepsilon \rightarrow 0+} \Lambda_{|K+L|}(\varepsilon) \geqq$ $\lim _{\varepsilon \rightarrow 0+} \Lambda_{|K|}(q+\varepsilon)$ by Corollary 3. If $m\left(P_{|K|}[\mu, \infty)\right)>q$, then $\lim _{\varepsilon \rightarrow 0+} \Lambda_{|K|}(q+\varepsilon)=\mu \geqq\left\|K P_{K}(-\mu, \mu)\right\|_{\infty}$. If $m\left(P_{|K|}[\mu, \infty)\right)=q$, then $\Lambda_{|K|}(q+\varepsilon)=\Lambda_{\left|K P_{K}(-\mu, \mu)\right|}(\varepsilon)$ for all $\varepsilon>0$ and the result is immediate. If $m\left(P_{|K|}[\mu, \infty)\right)<q$, then $m\left(P_{|K|}(\mu-\varepsilon, \mu)\right)=\infty$ for every $\varepsilon>0$, so that $\lim _{\varepsilon \rightarrow 0+} \Lambda_{|K|}(q+\varepsilon)=\mu=\left\|K P_{K}(-\mu, \mu)\right\|_{\infty}$.

Now let $1 \leqq p<\infty$. Since the theorem is trivial for $\mu=0$, assume $\mu>0$. If $m\left(P_{|K+L|}[\lambda, \infty)\right)=\infty$ for some $\lambda>0$, then $\|K+L\|_{p}=\infty$ and the theorem holds trivially. Therefore, assume $m\left(P_{|K+L|}[\lambda, \infty)\right)<\infty$ for all $\lambda>0$. Fix $\lambda, 0<\lambda<\mu$. Let $\gamma=m\left(P_{|K+L|}[\lambda, \infty)\right)$ and let $\varepsilon>0$. Note that if $\lambda=\Lambda_{|K+L|}(\gamma+\varepsilon)$, then $m\left(P_{|K+L|}\left(\lambda-\varepsilon_{1}, \lambda\right)\right)=\infty$ for every $\varepsilon_{1}>0$. Therefore, we can assume $\lambda>\Lambda_{|K+L|}(\gamma+\varepsilon)$. By Corollary $3, \Lambda_{|K+L|}(\gamma+\varepsilon) \geqq \Lambda_{|K|}(\gamma+q+\varepsilon)$. Therefore, $m\left(P_{|K|}[\lambda, \infty)\right) \leqq$ $\gamma+q+\varepsilon$. Since $\varepsilon$ is arbitrary, $m\left(P_{|K+L|}[\lambda, \infty)\right) \geqq m\left(P_{|K|}[\lambda, \infty)\right)-q \geqq$ $m\left(P_{|K|}[\lambda, \mu)\right)$ for $\lambda<\mu$. Now $\|K+L\|_{p}^{p}=\int_{-\infty}^{\infty}|\lambda|^{p} d m\left(P_{K+L}(\lambda)\right)=$ $\int_{0}^{\infty} \lambda^{p} d m\left(P_{|K+L|}(\lambda)\right)$ and

$$
\left\|K P_{K}(-\mu, \mu)\right\|_{p}^{p}=\int_{-\mu<\lambda<\mu}|\lambda|^{p} d m\left(P_{K}(\lambda)\right)=\int_{0 \leqq \lambda<\mu} \lambda^{p} d m\left(P_{|K|}(\lambda)\right) .
$$

The conclusion follows immediately. 


\section{REFERENCES}

1. J. Bendat and S. Sherman, Monotone and convex operator functions, Trans. Amer. Math. Soc., 79 (1955), 58-71.

2. J. Dixmier, Les Algèbres D'opérateurs dans L'espace Hilbertien, $2^{\text {ed }}$ edition, Paris, Gauthier-Villars, 1969.

3. R. A. Kunze, $L_{p}$ Fourier transforms on locally compact unimodular groups, Trans. Amer. Math. Soc., 89 (1958), 519-540.

4. J. von Neumann, Collected Works, Volume III, New York, MacMillan, 1961.

5. I. E. Segal, $A$ noncommutative extension of abstract integration, Ann. of Math., 57 (1953), 401-457.

6. H. Weyl, Das asymptotische Verteilungsgesetz der Eigenwerte linearer partieller Differentialgleichungen, Math. Ann., 71 (1912), 441-479.

Received February 12, 1973.

Cleveland State University 


\section{PACIFIC JOURNAL OF MATHEMATICS}

\section{EDITORS}

RICHARD ARENS (Managing Editor)

University of California

Los Angeles, California 90024

R. A. Beaumont

University of Washington

Seattle, Washington 98105
J. DugundJI

Department of Mathematics

University of Southern California

Los Angeles, California 90007

D. Gilbarg and J. Milgram

Stanford University

Stanford, California 94305

\section{ASSOCIATE EDITORS}

E. F. BECKENBACH

B. H. NeUMANN

F. WOLF

K. YosHIDA

\section{SUPPORTING INSTITUTIONS}

UNIVERSITY OF BRITISH COLUMBIA

CALIFORNIA INSTITUTE OF TECHNOLOGY

UNIVERSITY OF CALIFORNIA

MONTANA STATE UNIVERSITY

UNIVERSITY OF NEVADA

NEW MEXICO STATE UNIVERSITY

OREGON STATE UNIVERSITY

UNIVERSITY OF OREGON

OSAKA UNIVERSITY
UNIVERSITY OF SOUTHERN CALIFORNIA

STANFORD UNIVERSITY

UNIVERSITY OF TOKYO

UNIVERSITY OF UTAH

WASHINGTON STATE UNIVERSITY

UNIVERSITY OF WASHINGTON

$\stackrel{*}{*} \stackrel{*}{*} \stackrel{*}{*}$ AMERICAN MATHEMATICAL SOCIETY




\section{Pacific Journal of Mathematics}

\section{Vol. 53, No. $1 \quad$ March, 1974}

Martin Bartelt, Strongly unique best approximates to a function on a set, and a finite

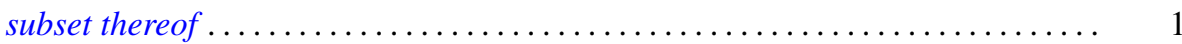

S. J. Bernau, Theorems of Korovkin type for $L_{p}$-spaces $\ldots \ldots \ldots \ldots \ldots \ldots \ldots \ldots \ldots$

S. J. Bernau and Howard E. Lacey, The range of a contractive projection on an

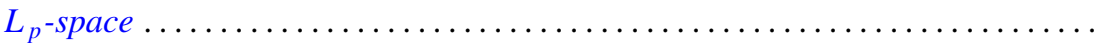

Marilyn Breen, Decomposition theorems for 3-convex subsets of the plane ......... Ronald Elroy Bruck, Jr., A common fixed point theorem for a commuting family of

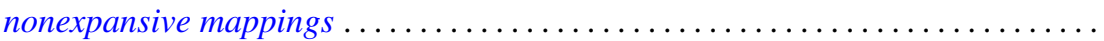

Aiden A. Bruen and J. C. Fisher, Blocking sets and complete $k$-arcs . . . . . . . 73

R. Creighton Buck, Approximation properties of vector valued functions . ......... 85

Mary Rodriguez Embry and Marvin Rosenblum, Spectra, tensor products, and

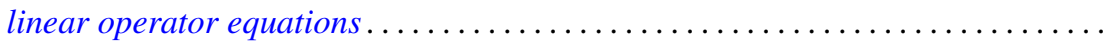

Edward William Formanek, Maximal quotient rings of group rings . . . . . . . . . 109

Barry J. Gardner, Some aspects of T-nilpotence . . . . . . . . . . . . . . . 117

Juan A. Gatica and William A. Kirk, A fixed point theorem for $k$-set-contractions

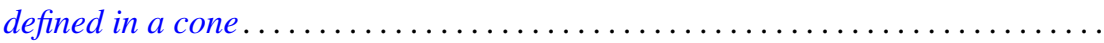

Kenneth R. Goodearl, Localization and splitting in hereditary noetherian prime

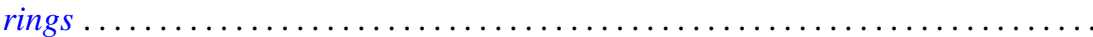

James Victor Herod, Generators for evolution systems with quasi continuous

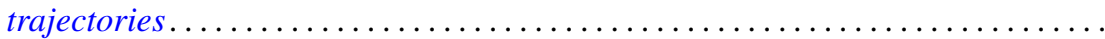

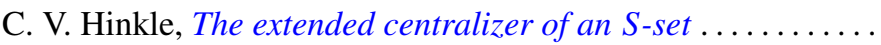

I. Martin (Irving) Isaacs, Lifting Brauer characters of p-solvable groups . . .

Bruce R. Johnson, Generalized Lerch zeta function ...........

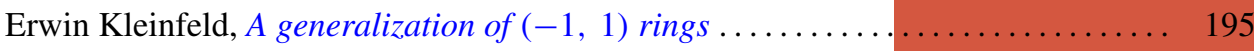

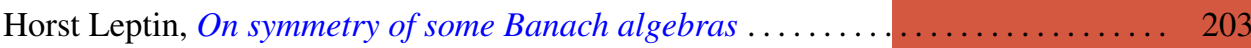

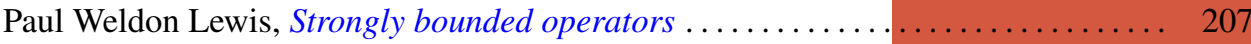

Arthur Larry Lieberman, Spectral distribution of the sum of self-adjoint

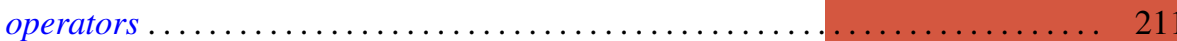

I. J. Maddox and Michael A. L. Willey, Continuous operators on paranormed spaces and matrix transformations

James Dolan Reid, On rings on groups ........................... 229

Richard Miles Schori and James Edward West, Hyperspaces of graphs are Hilbert cubes.

William H. Specht, A factorization theorem for p-constrained groups ...

Robert L Thele, Iterative techniques for approximation of fixed points of certain nonlinear mappings in Banach spaces ...............

Tim Eden Traynor, An elementary proof of the lifting theorem

Charles Irvin Vinsonhaler and William Jennings Wickless, Completely decomposable groups which admit only nilpotent multiplications .

Raymond O’Neil Wells, Jr, Comparison of de Rham and Dolbeault cohomology for

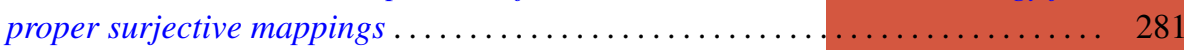

David Lee Wright, The non-minimality of induced central representations . . . . . 301 